05,11

\title{
Спиральное магнитное упорядочение и переход металл-диэлектрик в модели Хаббарда на треугольной решетке
}

\author{
(C) В.Ф. Гильмутдинов, М.А. Тимиргазин, А.К. Аржников \\ Удмуртский федеральный исследовательский центр УрО РАН, \\ Ижевск, Россия \\ E-mail: vital@udman.ru
}

Поступила в Редакцию 18 августа 2021 г.

В окончательной редакции 18 августа 2021 г.

Принята к публикации 4 сентября 2021 г.

\begin{abstract}
Построены магнитные фазовые диаграммы двумерной модели Хаббарда для изотропной и анизотропной треугольных решеток. Использовались приближения Хартри-Фока и вспомогательных бозонов. Показано, что наряду с коллинеарными магнитными состояниями (страйповое антиферромагнитное, ферромагнитное) в значительном интервале параметров модели реализуются неколлинеарные и спиральные магнитные состояния, характерные для треугольной решетки, а также фазовое расслоение между ними. Обнаружены фазовые переходы первого и второго рода, определены границы областей фазового расслоения. Сравнение двух приближений, Хартри-Фока и вспомогательных бозонов, показывает, что электронные корреляции подавляют магнитные состояния, расширяя область парамагнетизма, для значений $U / t \gtrsim 5$. В то же время, при концентрациях электронов с уровнем Ферми вблизи особенности ван Хова корреляции не вносят качественных изменений в диаграммы, что согласуется с ранее полученным результатом для квадратной и кубических решеток. Проведено сравнение результатов с имеющимися в литературе данными для других методов и подходов.
\end{abstract}

Ключевые слова: модель Хаббарда, фазовое расслоение, спиральное магнитное упорядочение, треугольная решетка, переход металл-изолятор.

DOI: 10.21883/FTT.2022.01.51835.191

\section{1. Введение}

Особый интерес в изучении магнитных свойств сильно коррелированных электронных систем представляют соединения с фрустрированными магнитными состояниями. К ним относятся, например, органические сверхпроводники (в основе которых лежат молекулы BEDT-TTF), кобальтиты натрия $\mathrm{Na}_{2} \mathrm{CoO}_{x}$ и др. Димеры молекул BEDT-TTF имеют по одному локализованному электрону и эффективно образуют слои треугольных решеток, между которыми расположены анионы, что обуславливает квазидвумерную проводимость [1]. Из-за явления геометрической фрустрации в таком типе решеток невозможно формирование шахматного (неелевского) антиферромагнитного (АФ) упорядочения, но стабилизируются другие магнитные структуры, не характерные для систем с квадратной и кубическими решетками. Андерсон в работе [2] показал, что основное состояние в треугольной решетке может представлять квантовомеханическую суперпозицию синглетных пар, заполняющих решетку. Это новое состояние материи, которое было названо,спиновой жидкостью“, обладает уникальными свойствами, в частности, возбужденные состояния могут представлять собой спиноны - магнитные объекты, обладающие нулевым электрическим зарядом.

Данные экспериментальных исследований $[3,4]$ указывают на формирование в системах с треугольной решеткой как коллинеарного АФ, так и (несоизмеримых) неколлинеарных магнитных конфигураций. Теоретические расчеты в рамках модели Хаббарда, которая традиционно используется для описания магнетизма и сверхпроводимости сильно коррелированных электронных систем, также предсказывают формирование разнообразных магнитных состояний.

Магнитные фазовые диаграммы (МФД) модели Хаббарда для анизотропной треугольной решетки в неограниченном методе Хартри-Фока (ХФ) (учитываются как зарядовые, так и спиновые флуктуации с некоторым весовым множителем) были построены в работе [5]. Рассматривались коллинеарные ферромагнитное (ФМ) и страйповое АФ-упорядочения, а также компланарные и некомпланарные спиральные фазы. Исследование показало стабилизацию неколлинеарных магнитных состояний в широкой области параметров модели, а также магнитное фазовое расслоение (ФР) между ними.

Учет электронных корреляций с помощью приближения вспомогательных бозонов (ВБ) Котляра и Рукенштайна для модели Хаббарда был выполнен в работах [6] и [7]. Авторами статьи [6] была построена МФД модели Хаббарда с учетом АФ, парамагнитного (ПМ) состояний и линейно поляризованной волны спиновой плотности. Был обнаружен фазовый переход первого рода между АФ- и ПМ-состояниями. В работе [7] была построена МФД-модели Хаббарда с учетом зарядового и спинового упорядочения и электронного переноса в пределах 


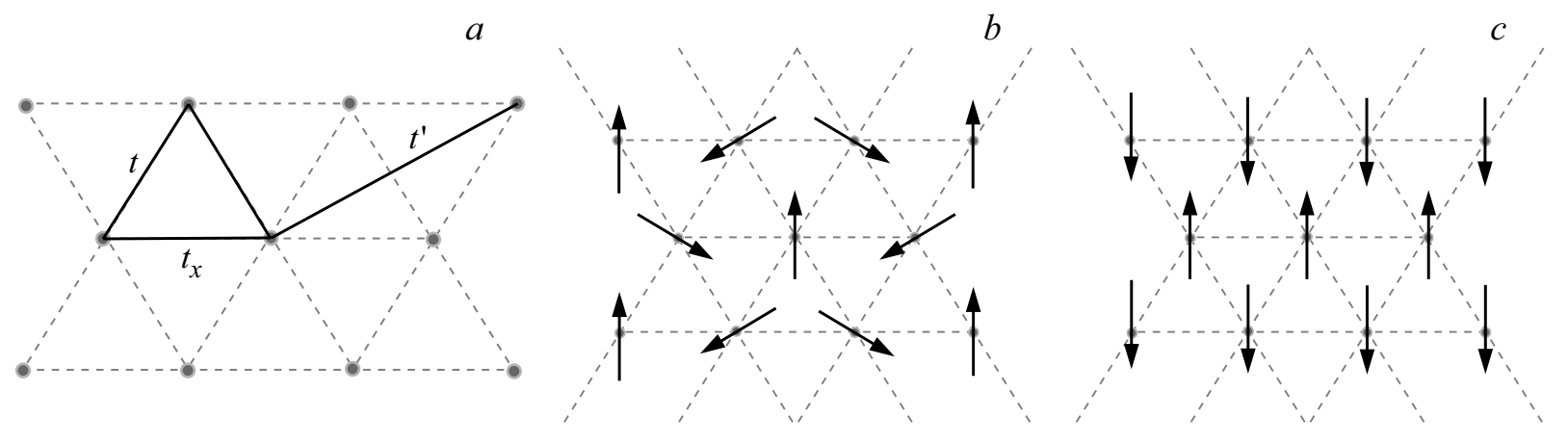

Рис. 1. a) Интегралы переноса в системе с треугольной решеткой, $b$ ) структура $\left.120^{\circ}, c\right)$ страйповое упорядочение.

первых трех координационных сфер. Были обнаружены фазовые переходы первого и второго рода между различными магнитными фазами. Оба вышеупомянутых исследования представлены в ограниченном диапазоне параметров модели и не учитывают всевозможные спиральные магнитные состояния с переменным волновым вектором.

Отдельное направление исследований связано с изучением перехода металл-диэлектрик (ПМД), происходящем при половинном заполнении с ростом параметра взаимодействия. При этом особое внимание уделяется поиску области стабильного немагнитного диэлектрика, указывающего на возможность формирования спиновой жидкости [8]. Ранее ПМД рассматривался в рамках вариационного кластерного приближения (VCA) $[9,10]$, неограниченного метода Хартри-Фока (UHF) [5], функционала ренормгруппы (fRG) [11-13], ячеечного метода динамического среднего поля (DMFT) [14], метода Монте-Карло [15], комбинации UHF и вариационного Монте-Карло [16]. В рамках приближения ВБ было показано [17], что, в отличие от случая квадратной решетки, где идеальный нестинг поверхности Ферми приводит к ПМД- и АФ-упорядочению при сколь угодно малых значениях хаббардовского параметра взаимодействия $U$, на треугольной решетке из-за отсутствия идеального нестинга переход происходит при конечном значении $U$. В зависимости от выбранного подхода и параметров модели получены как магнитные ПМД с участием АФ и различных неколлинеарных магнитных состояний, так и моттовский парамагнитный ПМД. В работе [10] отмечалось появление состояния спиновой жидкости вблизи значения интеграла электронного переноса, соответствующего соединениям BEDT-TTF, в режиме слабых корреляций $4 \lesssim U / t \lesssim 10$.

Несмотря на то, что изучению магнитных свойств квазидвумерных электронных систем с треугольной решеткой в течение долгого времени уделяется значительное внимание, до сих пор представленные в литературе данные являются неполными. В частности, ограничен диапазон используемых параметров моделей или рассматриваемых магнитных фаз [6], или пренебрегается учетом спиральных состояний с произвольным волновым вектором магнитной спирали [5], и др. До сих пор не проводились исследования, в которых последовательно учитывались бы произвольные волновые векторы магнитной спирали с одновременным сравнением результатов методов ХФ и ВБ. Такое сравнение позволило бы определить роль электронных корреляций в формировании магнитных состояний в электронных системах с треугольной решеткой. В то же время такой подход успешно использовался для описания магнитных свойств систем с кубическими и квадратными решетками. Ранее в приближении вспомогательных бозонов для модели Хаббарда были изучены условия стабилизации спиральных магнитных состояний в работе [18], где были построены МФД с учетом возможности ФР. Также данный подход успешно применялся и для описания магнитных состояний на фрустрированной гранецентрированной кубической решетке [19].

\section{2. Формализм}

Мы рассматриваем основное состояние однозонной модели Хаббарда на треугольной решетке. Гамильтониан модели в узельном представлении имеет вид

$$
H=\sum_{j, j^{\prime}, \sigma} t_{j, j^{\prime}} c_{j, \sigma}^{\dagger} c_{j^{\prime}, \sigma}-\mu \sum_{j, \sigma} c_{j, \sigma}^{\dagger} c_{j, \sigma}+U \sum_{j} n_{j, \uparrow} n_{j, \downarrow}
$$

где $t_{j, j^{\prime}}$ - интеграл переноса электронов с узла $j$ на узел $j^{\prime}, c_{j, \sigma}^{\dagger}\left(c_{j, \sigma}\right)$ - оператор рождения (уничтожения) электрона со спином $\sigma=(\uparrow, \downarrow)$ на узле $j, U-$ величина кулоновского взаимодействия электронов на узле $j$, $n_{j, \sigma}=c_{j, \sigma}^{\dagger} c_{j, \sigma}-$ оператор плотности электронов со спином $\sigma$ на узле $j, \mu-$ химический потенциал. Нами учитывается перескок электронов в пределах первой и второй координационных сфер с интегралами переноса $-t$ и $t^{\prime}$ соответственно, при этом вводилась анизотропия в выделенном направлении $x\left(t_{x} \neq t\right)$ (см. рис. 1, $\left.a\right)$. С учетом этого энергетический спектр свободных элект- 
ронов имеет вид

$$
\begin{aligned}
\varepsilon_{\mathbf{k}}= & -2 t_{x} \cos k_{x}-4 t \cos \frac{k_{x}}{2} \cos \frac{k_{y} \sqrt{3}}{2} \\
& +2 t^{\prime}\left(\cos k_{y} \sqrt{3}+2 \cos \frac{3 k_{x}}{2} \cos \frac{k_{y} \sqrt{3}}{2}\right) .
\end{aligned}
$$

Мы учитываем все многообразие спиральных магнитных состояний, при этом, как показывают расчеты, в основном состоянии реализуются следующие типы магнитного порядка: $120^{\circ}$-структура (см. рис. $1, b$ ) с $\mathbf{Q}=(0,4 \pi / 3)$, коллинеарное страйповое упорядочение (см. рис. $1, c)$ с $\mathbf{Q}=(0,2 \pi / \sqrt{3})$, а также спиральные магнитные состояния с волновыми векторами $\mathbf{Q}:(0, Q)$, $(Q, 0)$ и $(Q, 2 \pi / \sqrt{3})$.

Магнитное состояние на узле $j$ может быть описано следующим вектором магнитного момента

$$
\mathbf{m}_{j}=m\left(\cos \mathbf{Q} \mathbf{R}_{j}, \sin \mathbf{Q R} j\right),
$$

где $m$ - амплитуда магнитного момента, $\mathbf{R}_{j}-$ радиусвектор узла $j$.

Операция преобразования поворота $\mathrm{SU}(2)$ на угол $\mathbf{Q R}_{j}$ вокруг оси $z$ позволяет привести спиральные магнитные состояния к ФМ порядку.

$$
\widehat{A}\left(\mathbf{R}_{j}\right)=e^{i(\mathbf{n} \hat{\boldsymbol{\sigma}}) \frac{\mathbf{Q R}_{j}}{2}}=\sigma^{0} \cos \frac{\mathbf{Q R}}{2}+i(\mathbf{n} \widehat{\boldsymbol{\sigma}}) \sin \frac{\mathbf{Q R}}{2},
$$

где $\mathbf{R}_{j}$ - радиус-вектор узла $j, \mathbf{Q}-$ волновой вектор магнитной спирали, $\mathbf{n}$ - единичный вектор, направление которого соответствует направлению магнитного момента $m$ на узле, $\widehat{\sigma}^{0}$ - единичная матрица, $\widehat{\sigma}=\left(\sigma^{x}, \widehat{\sigma}^{y}, \widehat{\sigma}^{z}\right)-$ генераторы группы $\mathrm{SU}(2)$ (матрицы Паули).

\section{1. Метод Хартри-Фока}

После поворота и преобразования Фурье в приближении ХФ гамильтониан (1) принимает вид

$$
\begin{gathered}
H^{\mathrm{HFA}}=\sum_{\mathbf{k}, \sigma, \sigma^{\prime}} t_{\mathbf{k}}^{\sigma, \sigma^{\prime}} c_{\mathbf{k}, \sigma}^{\dagger} c_{\mathbf{k}, \sigma^{\prime}}-\mu \sum_{\mathbf{k}, \sigma} c_{\mathbf{k}, \sigma}^{\dagger} c_{\mathbf{k}, \sigma} \\
+\frac{N U}{4}\left(m^{2}-n^{2}\right)+\frac{U n}{2} \sum_{\mathbf{k}, \sigma} c_{\mathbf{k}, \sigma}^{\dagger} c_{\mathbf{k}, \sigma}-\frac{U m}{2} \sum_{\mathbf{k}, \sigma} \sigma c_{\mathbf{k}, \sigma}^{\dagger} c_{\mathbf{k}, \sigma} \\
t_{\mathbf{k}}^{\sigma, \sigma^{\prime}}=e_{\mathbf{k}}^{s} \delta_{\sigma, \sigma^{\prime}}+e_{\mathbf{k}}^{a} \delta_{\sigma, \sigma^{\prime}} \\
e_{\mathbf{k}}^{s}=\frac{\varepsilon_{\mathbf{k}+\frac{\mathbf{Q}}{2}}^{0}+\varepsilon_{\mathbf{k}-\frac{\mathbf{Q}}{2}}^{0}}{2} \\
e_{\mathbf{k}}^{a}=\frac{\varepsilon_{\mathbf{k}+\frac{\mathbf{Q}}{2}}^{0}-\varepsilon_{\mathbf{k}-\frac{\mathbf{Q}}{2}}^{0}}{2} .
\end{gathered}
$$

Здесь $N-$ полное число частиц в системе, $n=\left\langle\sum_{\mathbf{k}, \sigma} c_{\mathbf{k}, \sigma}^{\dagger} c_{\mathbf{k}, \sigma}\right\rangle$ и $m=\left\langle\sum_{\mathbf{k}, \sigma} \sigma c_{\mathbf{k}, \sigma}^{\dagger} c_{\mathbf{k}, \sigma}\right\rangle-$ средние значения электронной плотности и магнитного момента соответственно. Состояние системы определяется минимизацией термодинамического потенциала $\Omega_{\mathrm{HFA}}=\left\langle H^{\mathrm{HFA}}\right\rangle$ для каждой конфигурации параметров системы $\Omega_{\mathrm{HFA}}=\operatorname{argmin} \Omega_{\mathrm{HFA}}(\mu, \mathbf{Q}, U)(\langle\ldots\rangle$ означает квантовомеханическое усреднение по основному состоянию гамильтониана).

\section{2. Метод вспомогательных бозонов}

Мы используем метод ВБ в приближении седловой точки в трактовке Котляра и Рукенштайна [20], формализм которого достаточно подробно описан, например, в работе [18]. После преобразования поворота (4) вводятся вспомогательные бозонные операторы $e_{j}, p_{j, \sigma}$ и $d_{j}$, соответствующие пустому, занятому одним и двумя электронами узлу $j$, причем накладываются ограничения, исключающие нефизичные состояния:

$$
\begin{gathered}
e_{j}^{\dagger} e_{j}+\sum_{\sigma} p_{j, \sigma}^{\dagger} p_{j, \sigma}+d_{j}^{\dagger} d_{j}=1, \\
p_{j, \sigma}^{\dagger} p_{j, \sigma}+d_{j}^{\dagger} d_{j}=c_{j, \sigma}^{\dagger} c_{j, \sigma} .
\end{gathered}
$$

В трактовке Котляра и Рукенштайна проводится замена, обеспечивающая когерентность бозонных и фермионных полей: $c_{j, \sigma} \rightarrow z_{j, \sigma} f_{j, \sigma}$, где

$$
\begin{aligned}
& z_{j, \sigma}=\left(1-d_{j}^{\dagger} d_{j}-p_{j, \sigma}^{\dagger} p_{j, \sigma}\right)^{-1 / 2} \\
& \times\left(e_{j}^{\dagger} p_{j, \sigma}+p_{j, \sigma^{\prime}}^{\dagger} d_{j}\right)\left(1-d_{j}^{\dagger} d_{j}-p_{j, \sigma^{\prime}}^{\dagger} p_{j, \sigma^{\prime}}\right)^{-1 / 2} .
\end{aligned}
$$

Слагаемое $e_{j}^{\dagger} p_{j, \sigma}$ соответствует переходу из однократно занятого состояния в пустое, а $p_{j, \sigma^{\prime}}^{\dagger} d_{j \sigma}-$ переходу из двукратно занятого состояния в однократное. Во введенной параметризации гамильтониан приобретает диагональный по отношению к бозонным операторам вид

$$
H^{\mathrm{SBA}}=\sum_{j, j^{\prime}, \sigma, \sigma^{\prime}} t_{j, j^{\prime}}^{\sigma, \sigma^{\prime}} c_{j, \sigma^{\prime}}^{\dagger} c_{j^{\prime}, \sigma^{\prime}} z_{j, \sigma}^{\dagger} z_{j^{\prime}, \sigma^{\prime}}+U \sum_{j} d_{j}^{\dagger} d_{j} .
$$

В статическом приближении термодинамический потенциал большого канонического ансамбля системы имеет вид

$$
\begin{aligned}
\Omega_{\mathrm{SBA}}= & \eta\left(e^{2}+p_{\uparrow}^{2}+p_{\downarrow}^{2}+d^{2}-1\right) \\
& +U d^{2}-\sum_{\sigma} \lambda_{\sigma}\left(p_{\sigma}^{2}+d^{2}\right)+\Omega_{f} .
\end{aligned}
$$

Здесь $\lambda_{\sigma}$ и $\eta-$ множители Лагранжа, а $\Omega_{f}-$ стандартный термодинамический потенциал фермионной системы. Минимизация термодинамического потенциала по отношению к волновому вектору $\mathbf{Q}$ позволяет определить основное магнитное состояние системы при заданных параметрах $(U / t, n)$ и построить МФД-модели. 


\section{3. Результаты}

\section{1. Диаграммы $(U / t, n)$}

Результаты исследований представлены на рис. 2 и 3 соответственно. МФД показывают, что в системе реализуются как коллинеарные (страйповое, ФМ), так и спиральные $\left(120^{\circ}\right.$-структура, фазы с переменным волновым вектором $(0, Q),(Q, 0)$, и $(Q, 2 \pi / \sqrt{3}))$ магнитные состояния. При построении данных диаграмм мы не учитывали анизотропию интеграла электронного переноса и перескоки на вторых (следующих за ближайшими) соседей.

В широкой области параметров диаграммы реализуется ФР между различными состояниями. Для учета возможности ФР в качестве базовой переменной используется химический потенциал $\mu$, а количество электронов $n$ фигурирует в виде параметра. Фазовый переход первого рода, сопроводжаемый ФР, характеризуется скачком по параметрам магнитной структуры $\mathbf{Q}$ и $m$ : из $\left(\mathbf{Q}_{1}, m_{1}\right)$ в $\left(\mathbf{Q}_{2}, m_{2}\right)$. Этот переход в переменных $(U, \mu)$ характеризуется также скачком $\Delta(n)=\left|n\left(\mu, \mathbf{Q}_{1}, m_{1}\right)-n\left(\mu, \mathbf{Q}_{2}, m_{2}\right)\right|$. Если $n$ лежит между $n\left(\mu, \mathbf{Q}_{1}, m_{1}\right)$ и $n\left(\mu, \mathbf{Q}_{2}, m_{2}\right)$, в системе одновременно сосуществуют две пространственно расслоенные фазы с плотностями $n\left(\mu, \mathbf{Q}_{1}, m_{1}\right)$ и $n\left(\mu, \mathbf{Q}_{2}, m_{2}\right)$, определяющими левую и правую границы области ФР на МФД [21].

Сушествует ряд отличий между диаграммами для методов ХФ и ВБ. Применение метода ВБ приводит к подавлению магнитного порядка сильными электронными корреляциями, и область упорядоченных фаз сужается к линии половинного заполнения. Для $U / t>5$ в значительной мере сокращается многообразие спиральных магнитных фаз: из них реализуется только состояние $(Q, 0)$ в части диаграммы, соответствующей дырочному допированию $n>1$, а при $n<1$ учет сильных электронных корреляций приводит к полному исчезновению страйпового, спирального и ФМ-состояний. При $U / t<5$ многообразие спиральных состояний сохраняется, но вид диаграммы меняется на количественном уровне: ПМ-область значительно расширяется. Кроме того, в методе ВБ для значений $U / t>5$ появляются области ФР между ПМ- и магнитными состояниями (со $120^{\circ}$-состоянием в области допирования дырками и с $Ф \mathrm{M} \mathrm{-} \mathrm{в} \mathrm{области} \mathrm{допирования} \mathrm{электронами).} \mathrm{Для}$ диаграммы, построенной в приближении ХФ, при указанных значениях $U / t$ все переходы в ПМ состояние являются фазовыми переходами второго рода и не сопровождаются расслоением фаз.

С ростом $U / t$ в части диаграммы, соответствующей допированию электронами $n>1$, расширяется область ФМ-состояния. Это согласуется с теоремой Нагаока, которая утверждает, что на треугольной решетке в пределе $U \rightarrow \infty$ при допировании одним электроном (что эквивалентно допированию дыркой при $t>0$ ) основным состоянием модели Хаббарда является насыщенный ферромагнетизм [22,23]. Аналогичный результат ранее

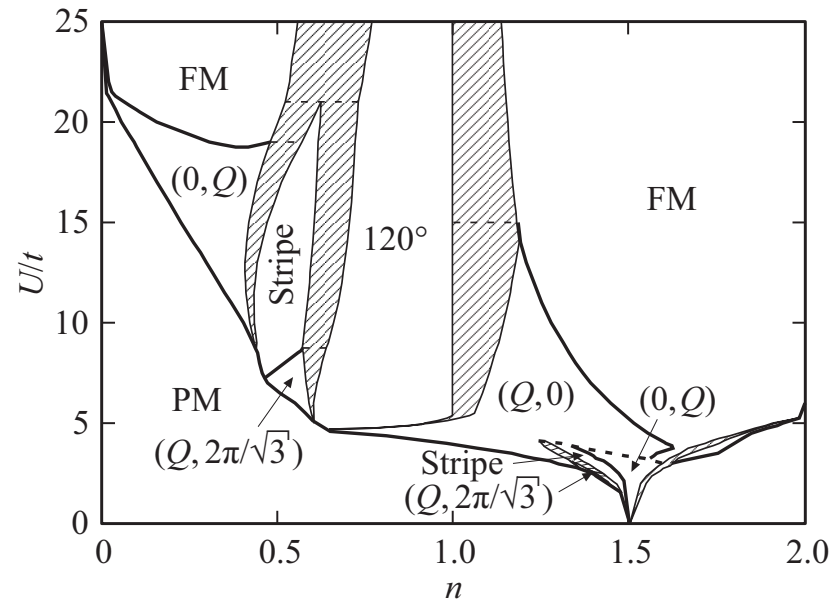

Рис. 2. Магнитная фазовая диаграмма основного состояния модели Хаббарда для треугольной решетки, построенная в приближении ХФ. Жирными линиями обозначены границы фазовых переходов второго рода, тонкие линии разделяют области однородной фазы и фазового расслоения (заштрихованные области). Тонкие пунктирные горизонтальные линии разделяют области фазового расслоения для разных пар фаз. Жирными пунктирными линиями обозначены фазовые переходы первого рода. Аббревиатурами „РМ“, „FМ“ и „Stripe“ обозначены области парамагнитного, ферромагнитного и страйпового состояний соответственно. Области спирального спинового упорядочения с переменным волновым вектором обозначены с указанием в скобках проекций волнового вектора магнитной спирали.

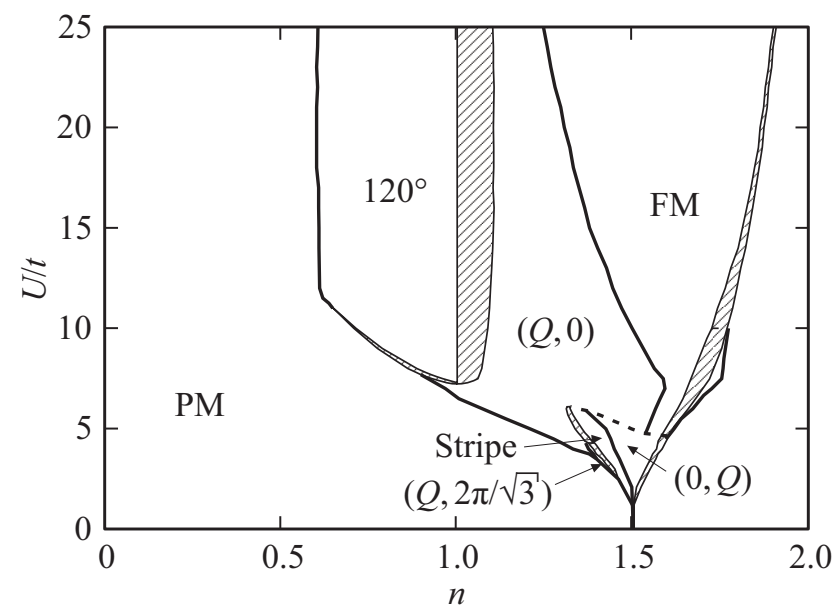

Рис. 3. Магнитная фазовая диаграмма основного состояния модели Хаббарда для треугольной решетки, построенная в приближении ВБ. Обозначения те же, что и на рис. 2.

был получен для другой фрустрированной системы ГЦК-решетки [19].

В статье [5] рассматриваются некомпланарные состояния, однако, в отличие от нашей работы, не учитывается все многообразие спиральных состояний. Кроме того, в этой работе не обсуждается характер фазовых переходов между магнитными состояниями. Учет разных на- 
боров магнитоупорядоченных фаз и разница в применяемых подходах приводит к качественным и количественным различиям. В дырочно-допированной области нашей диаграммы страйповое состояние существует в пределах $6 \lesssim U / t \lesssim 21$, тогда как в работе [5] оно не реализуется в отсутствие анизотропии интеграла электронного переноса и появляется лишь с ростом величины анизотропии в узкой области параметра $10 \lesssim U / t \lesssim 15$. В области допирования дырками на наших диаграммах при $U / t>15$ ФМ-состояние претерпевает фазовое расслоение со $120^{\circ}$-структурой. В исследовании [5] фазовое расслоение в этой же области реализуется с конической спиральной структурой, за которой следует ферромагнитная фаза. В то же время, в интервале параметров $0.6 \lesssim n \lesssim 1$ и $5 \lesssim U / t \lesssim 15$ и $1.4 \lesssim n \lesssim 2.0$ и $5 \lesssim U / t \lesssim 15$ наши результаты совпадают с результатами работы [5].

\section{2. Переход металл-диэлектрик}

На рис. 4 и 5 представлены МФД в координатах $\left(t^{\prime} / t, U / t\right)$ при $n=1$, построенные с помощью метода ВБ, для двух случаев: 1) изотропного электронного переноса $t$ с учетом перескоков на вторые соседние узлы $t^{\prime}$ и 2) с анизотропией электронного переноса $\left(t, t_{x}\right.$; см. рис. 1). На диаграммах присутствуют области металлического состояния с ПМ- и спиральным магнитным упорядочением, а также диэлектрика со страйповым, спиральным и $120^{\circ}$-магнитным упорядочением. Переходы между состояниями с постоянным волновым вектором и переход из металлического в диэлектрическое состояние являются фазовыми переходами первого рода. Фазовыми переходами второго рода являются переходы из состояния страйпового магнитного в спиральное диэлектрическое, а также переходы из ПМ в спиральное металлическое. Дополнительно на обеих диаграммах (вставки) изображены переходы из ПМ-металлического состояния в ПМ-диэлектрическое (ПД). Это фазовый переход второго рода, который для изотропного случая происходит при $U / t>16$, для анизотропного - при $U / t>13$. На МФД видно, что при таких высоких значениях $U / t$ стабилизируются магнитные состояния таким образом, ПД-состояние не является основным, а значит, спиновая жидкость в нашем подходе не обнаруживается.

Ранее ПМД на треугольной решетке был рассмотрен с помощью метода VCA в работе [9], где было получено значительно более низкое критическое значение параметра взаимодействия $U / t \sim 4$, что позволяло авторам утверждать о формировании состояния спиновой жидкости. В $[10,11]$ построена МФД модели Хаббарда в координатах $\left(U / t, t_{x} / t\right)$ при $n=1$ в рамках метода вариационного кластерного приближения и метода ренормгруппы. В области параметров $5 \lesssim U / t \lesssim 9$ и $0.8 \lesssim n \lesssim 1$ ими было получено состояние немагнитного изолятора. Ограничением данных работ является то, что в них не учитывалось состояние спиновой спирали, которое в наших расчетах является основным состоянием.

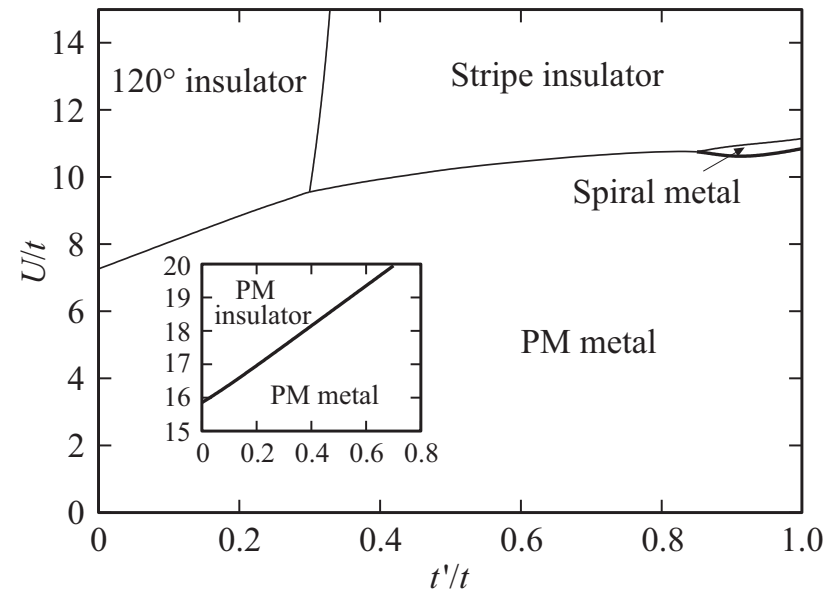

Рис. 4. Фазовая диаграмма модели Хаббарда в приближении ВБ для $n=1$ с учетом электронного переноса на первую $t$ и вторую $t^{\prime}$ кординационные сферы. Жирными линиями изображены фазовые переходы второго рода, тонкими - первого. Области „spiral metal“ соответствует волновой вектор $(Q, 2 \pi / \sqrt{3})$. На вставке изображена аналогичная фазовая диаграмма, построенная без учета магнитных состояний.

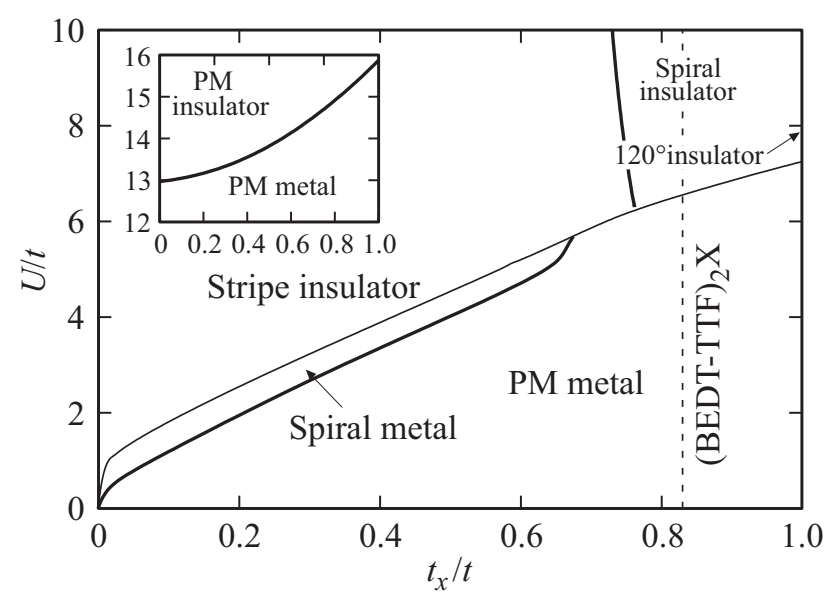

Рис. 5. Фазовая диаграмма модели Хаббарда в приближении вспомогательных бозонов в переменных $\left(t_{x} / t, U / t\right)$ с учетом анизотропии интеграла электронного переноса по оси $x$. Вертикальной пунктирной линией обозначена величина $t_{x} / t$, соответствующая органическим сверхпроводникам BEDT-TTF. Остальные обозначения те же, что и на рис. 4. На вставке изображена аналогичная фазовая диаграмма, построенная без учета магнитных состояний.

На диаграмме 5 нанесена вертикальная пунктирная линия при значении $t_{x} / t=0.83$, соответствующем органическим сверхпроводникам на основе BEDT-TTF [16]. Из анализа диаграммы, таким образом, можно сделать вывод, что наше исследование предсказывает для этого класса соединений переход первого рода из ПМ-металлического состояния в магнитное диэлектрическое со спиральной спиновой структурой при повышении параметра $U / t$. 


\section{4. Заключение}

В настоящей работе мы изучили условия формирования магнитных состояний на изотропной и анизотропной треугольной решетке в модели Хаббарда. Использовались приближения ХФ и ВБ. Нами было показано, что, наряду с коллинеарными магнитными состояниями (ФМ, страйповое АФ), в системах с треугольной решеткой формируются также спиральные магнитные состояния с волновыми векторами $(0, Q)$, $(Q, 0)$ и $(Q, 2 \pi / \sqrt{3})$. Учет электронных корреляций приводит к расширению области ПМ состояния и снижению многообразия магнитных фаз, что согласуется с ранее полученными результатами для квадратной и кубической решеток [18]. Формирование $120^{\circ}$-магнитной структуры в области половинного заполнения и расширение концентрационной области существования ПМ при уменьшении параметра $U / t$ является характерным как для наших исследований, так и для работ других авторов. Существенным отличием нашего подхода является учет всего разнообразия спиральных магнитных состояний, а также областей фазового расслоения, занимающих значительную часть диаграмм. Следствием этого, в частности, является широкая область магнитных состояний на диаграммах перехода металл-диэлектрик, вытесняющая область магнитного изолятора.

\section{Финансирование работы}

Работа выполнена в рамках государственного задания Министерства науки и высшего образования Российской Федерации (тема № 121030100005-1).

\section{Конфликт интересов}

Авторы заявляют, что у них нет конфликта интересов.

\section{Список литературы}

[1] Y. Shimizu, K. Miyagawa, K. Kanoda, M. Maesato, G. Saito. Phys. Rev. Lett. 91, 10, 107001 (2003).

[2] P.W. Anderson. Mater. Res. Bull. 8, 2, 153 (1973).

[3] P. Limelette, P. Wzietek, S. Florens, A. Georges, T.A. Costi, C. Pasquier, D. Jérome, C. Mézière, P. Batail. Phys. Rev. Lett. 91, 1, 016401 (2003).

[4] H. Nakamura, T. Yamasaki, S. Giri, H. Imai, M. Shiga, K. Kojima, M. Nishi, K. Kakurai, N. Metoki. J. Phys. Soc. Jpn 69, 9, 2763 (2000).

[5] K. Pasrija, S. Kumar. Phys. Rev. B 93, 19, 195110 (2016).

[6] A. Feiguin, C. Gazza, A. Trumper, H. Ceatto. J. Phys.: Condens. Matter 9, 4, L27 (1999).

[7] K. Jiang, S. Zhou, Z. Wang. Phys. Rev. B 90, 16, 165135 (2014).

[8] T. Mizusaki, M. Imada. Phys. Rev. B 74, 1, 014421 (2006).

[9] K. Misumi, T. Kaneko, Y. Ohta. Phys. Rev. B 95, 7, 075124 (2017).

[10] M. Laubach, R. Thomale, C. Platt, W. Hanke, G. Li. Phys. Rev. B 91, 24, 245125 (2015).
[11] H. Morita, S. Watanabe, M. Imada. J. Phys. Soc. Jpn 71, 9, 2109 (2002).

[12] Z. Zhu, D.N. Sheng, A. Vishwanath. arXiv:2007.11963 (2020).

[13] A. Szasz, J. Motruk. arXiv:2101.07454 (2021).

[14] B. Kyung, A.-M.S. Tremblay. Phys. Rev. Lett. 97, 4, 046402 (2006).

[15] L. Tocchio, A. Montorsi, F. Becca. Phys. Rev. B 102, 11, 115150 (2020).

[16] L. Tocchio, H. Feldner, F. Becca, R. Valentí, C. Gros. Phys. Rev. B 87, 3, 035143 (2012).

[17] M. Capone, L. Capriotti, F. Becca, S. Caprara. Phys. Rev. B 63, 8, 085104 (2000).

[18] P.A. Igoshev, M.A. Timirgazin, V.F. Gilmutdinov, A.K. Arzhnikov, V.Y. Irkhin. J. Phys.: Condens. Matter 27, 44, 446002 (2015).

[19] M.A. Timirgazin, P.A. Igoshev, A.K. Arzhnikov, V.Y. Irkhin. J. Phys.: Condens. Matter 28, 50, 505601 (2016).

[20] G. Kotliar, A.E. Ruckenstein. Phys. Rev. Lett. 57, 11, 1362 (1986).

[21] P.A. Igoshev, M.A. Timirgazin, A.A. Katanin, A.K. Arzhnikov, V.Y. Irkhin. Phys. Rev. B 81, 9, 094407 (2010).

[22] Y. Nagaoka. Phys. Rev. 147, 1, 392 (1966).

[23] H. Tasaki. Phys. Rev. B 40, 13, 9192 (1989).

Редактор Е.В. Толстякова 